\title{
SMARCA4 inactivation defines a subset of undifferentiated uterine sarcomas with rhabdoid and small cell features and germline mutation association
}

Douglas I. Lin ${ }^{1, *}$, Justin M. Allen ${ }^{1}$, Jonathan L. Hecht ${ }^{2}$, Jonathan .K. Killian ${ }^{1}$, Nhu T. Ngo ${ }^{1}$, Claire Edgerly ${ }^{1}$, Eric A. Severson ${ }^{1}$, Siraj M. Ali ${ }^{1}$, Rachel L. Erlich ${ }^{1}$, Shakti H. Ramkissoon ${ }^{1,3}$, Jo-Anne Vergilio1, Jeffrey S. Ross ${ }^{1,4}$ and Julia A. Elvin ${ }^{1}$

1. Foundation Medicine Inc., Cambridge, MA. 2. Beth Israel Deaconess Medical Center, Boston, MA. 3. Wake Forest Comprehensive Cancer Center and Department of Pathology, Wake Forest School of Medicine, Winston-Salem, NC.

4. Upstate Medical University, Syracuse, NY.

A rare subset of aggressive SMARCA4-deficient uterine sarcomas has been recently proposed, with only a limited number of cases having been previously described. Here, we identify 16 additional cases of SMARCA4-deficient uterine sarcoma from the database of a large, CLIA-certified and CAPaccredited, reference molecular laboratory, and we expand on their clinicopathological and genomic features. Median patient age was 49 years (range 32-70). Most tumors were aggressive with distant metastasis. SMARCA4-deficient uterine sarcoma demonstrated predominantly rhabdoid or large epithelioid cells with abundant cytoplasm, but also had varying degrees of small cell and spindle cell morphology. Tumors were microsatellite stable and exhibited no other or only few co-occurring genomic alterations by comprehensive genomic profiling. We discovered one patient, who developed SMARCA4-deficient uterine sarcoma at age of 55, had a germline SMARCA4 mutation, whose daughter had previously died of small cell carcinoma of the ovary, hypercalcemic type, at the age of 32 . Our data support the notion that SMARCA4 inactivation is the driver oncogenic event of a morphologically and molecularly distinct form of uterine sarcoma. Identification of SMARCA4-deficient uterine sarcomas may be clinically important due to their aggressive behavior, germline association and emerging targeted therapies.

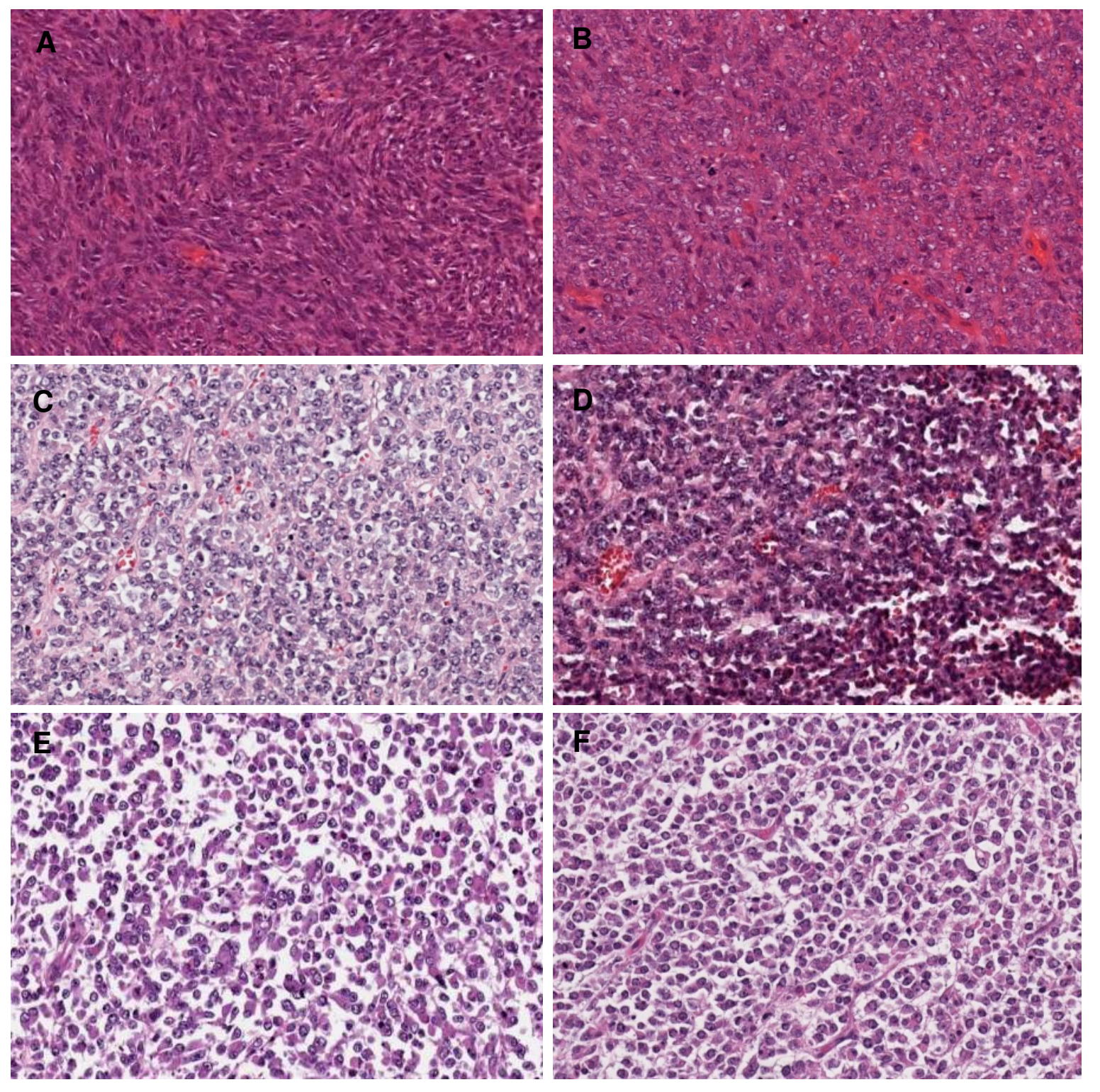

Figure 2. Morphological features of SMARCA4-deficient uterine sarcoma.

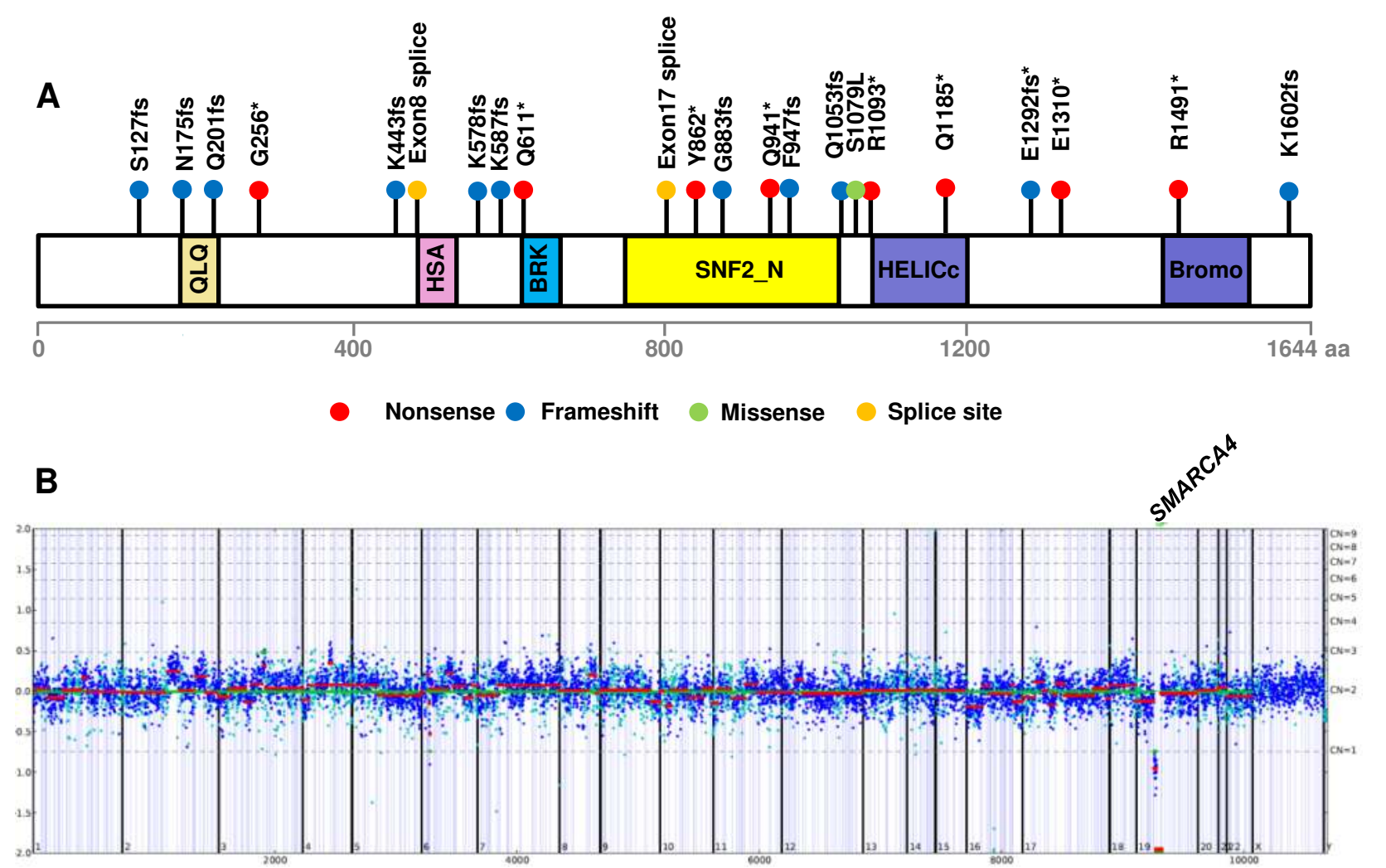

Figure 1. Mutational landscape of SMARCA4 across our SMARCA4-deficient uterine sarcoma cohort.

\begin{tabular}{|c|c|c|c|c|c|c|c|c|c|}
\hline $\mathrm{n}$ & Age & Submitting diagnosis & $\begin{array}{c}\text { Size } \\
(\mathrm{cm})\end{array}$ & Stage & LVI & LN & Ovary & Site & Procedure \\
\hline 1 & 48 & Undifferentiated sarcoma & 6 & IB & NA & NA & NA & Uterus & Biopsy \\
\hline 2 & 51 & Undifferentiated sarcoma & 14 & IIIC & Pos & Pos & Neg & Uterus & TLH BSO \\
\hline 3 & 63 & Undifferentiated sarcoma & 4 & IV & Neg & Neg & Neg & Uterus & TLH BSO \\
\hline 4 & 52 & Undifferentiated sarcoma & 16 & IV & Pos & Neg & Pos & Uterus & TLH BSO \\
\hline 5 & 43 & Undifferentiated sarcoma & Unk & IV & NA & NA & NA & Liver & Biopsy \\
\hline 6 & 51 & Undifferentiated sarcoma & 10.5 & IIIB & Pos & Neg & Neg & Uterus & TLH BSO \\
\hline 7 & 60 & Undifferentiated sarcoma & 12.9 & IV & Pos & NA & Neg & Uterus & TLH BSO \\
\hline 8 & 49 & Undifferentiated sarcoma & 6 & IIB & Pos & Neg & Pos & Uterus & TLH BSO \\
\hline 9 & 33 & Undifferentiated sarcoma & 6 & IVB & Pos & NA & Neg & Uterus & TLH BSO \\
\hline 10 & 70 & HG-ESS & 3.7 & IIIA & Neg & NA & Pos & Uterus & TLH BSO \\
\hline 11 & 52 & Undifferentiated neoplasm & 5.5 & IV & Pos & Pos & Neg & Uterus & TLH BSO \\
\hline 12 & 44 & Undifferentiated sarcoma & 6.9 & IIIC & Pos & Pos & Pos & Uterus & TLH BSO \\
\hline 13 & 38 & Undifferentiated sarcoma & 7 & IV & Pos & Pos & Neg & Uterus & TLH BSO \\
\hline 14 & 55 & Undifferentiated sarcoma & Unk & IV & NA & NA & Pos & Uterus & TLH BSO \\
\hline 15 & 45 & Undifferentiated sarcoma & 12.2 & IB & Pos & NA & Neg & Uterus & TLH BSO \\
\hline 16 & 32 & Uterine adenosarcoma & 17.7 & III & Pos & Pos & Neg & Uterus & TLH BSO \\
\hline
\end{tabular}

Table 1. Clinico-pathological features of SMARCA4-deficient uterine sarcoma.

\begin{tabular}{ll|l|l} 
SMARCA4 & $100 \%$ & Figure 3. Molecular features of \\
TP53 & $13 \%$ & $6 \%$ & $\begin{array}{l}\text { SMARCA4-deficient uterine } \\
\text { sarcoma following comprehensive } \\
\text { genomic profiling demonstrating }\end{array}$ \\
RB1 & $6 \%$ & $\begin{array}{l}\text { none or few co-alterations. Blue } \\
\text { rectangle: homozygous gene } \\
\text { deletion; black square: oncogenic } \\
\text { truncating mutation; green square: } \\
\text { oncogenic missense mutation, red } \\
\text { CTNNB1 }\end{array}$ \\
ZNF703 & $6 \%$ &
\end{tabular}
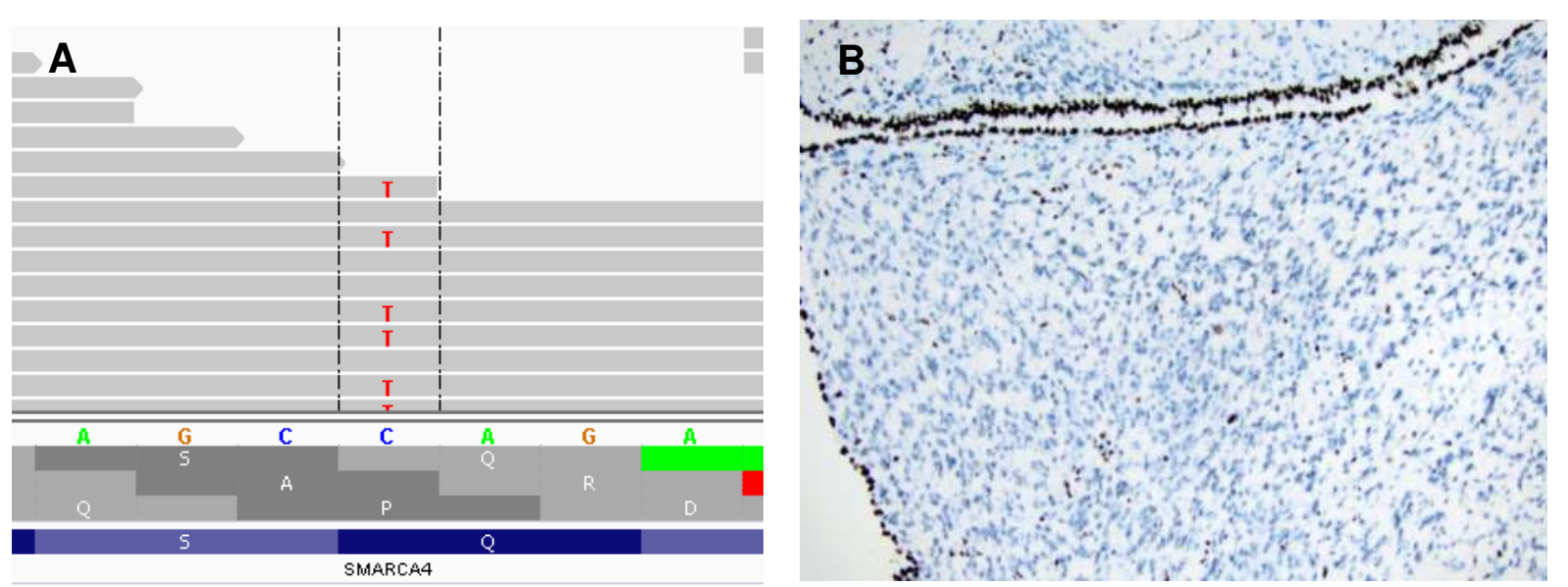

Figure 4. Case \#14 with germline SMARCA4 mutation. A. Next generation sequencing identified a 1831C>T (NM_003072), Q611* , nonsense tumor mutation that was further confirmed to be germline by genetic testing. (B) Immunohistochemistry of the patient's tumor revealed loss of BRG1 (protein encoded by SMARCA4) in tumor cells with appropriate labeling of benign endometrial epithelium and benign stromal cells (with BRG1 only) as internal control. 